\title{
Anisotropic intrinsic spin relaxation in graphene due to flexural distortions
}

\author{
S. Fratini, ${ }^{1}$ D. Gosálbez-Martínez, ${ }^{2}$ P. Merodio Cámara, ${ }^{1,3}$ and J. Fernández-Rossier ${ }^{4, *}$ \\ ${ }^{1}$ Institut Néel-CNRS and Université Joseph Fourier, Boîte Postale 166, F-38042 Grenoble Cedex 9, France \\ ${ }^{2}$ Departamento de Física Aplicada, Universidad de Alicante, 03690 San Vicente del Raspeig, Spain \\ ${ }^{3}$ SPINTEC, UMR CEA/CNRS/UJF-Grenoble I/Grenoble-INP, INAC, Grenoble, France \\ ${ }^{4}$ International Iberian Nanotechnology Laboratory (INL), Av. Mestre José Veiga, 4715-330 Braga, Portugal \\ (Received 7 March 2012; revised manuscript received 28 June 2013; published 20 September 2013)
}

\begin{abstract}
We propose an intrinsic spin scattering mechanism in graphene originated by the interplay of atomic spinorbit interaction and the local curvature induced by flexural distortions of the atomic lattice. Starting from a multiorbital tight-binding Hamiltonian with spin-orbit coupling considered nonperturbatively, we derive an effective Hamiltonian for the spin scattering of the Dirac electrons due to flexural distortions. We compute the spin lifetime due to both flexural phonons and ripples and we find values in the microsecond range at room temperature. Interestingly, this mechanism is anisotropic on two counts. First, the relaxation rate is different for off-plane and in-plane spin quantization axis. Second, the spin relaxation rate depends on the angle formed by the crystal momentum with the carbon-carbon bond. In addition, the spin lifetime is also valley dependent. The proposed mechanism sets an upper limit for spin lifetimes in graphene and will be relevant when samples of high quality can be fabricated free of extrinsic sources of spin relaxation.
\end{abstract}

DOI: 10.1103/PhysRevB.88.115426

PACS number(s): 73.61.Wp, 63.22.Rc, 72.25.Rb, 72.80.Vp

\section{INTRODUCTION}

The electron spin lifetime in carbon materials is expected to be very long both because of the very large natural abundance of the spinless nuclear isotope ${ }^{12} C$ and the small size of spinorbit coupling. In the case of flat graphene, the spin projection perpendicular to the plane is conserved, even in the presence of the intrinsic spin-orbit coupling. Thus graphene was proposed as an optimal material to store quantum information in the spin of confined electrons. ${ }^{1}$ Most of the experiments ${ }^{2-11}$ show that the spin lifetimes are in the range of nanoseconds, much shorter than expected from these considerations, which is being attributed to several extrinsic factors: the breaking of reflection symmetry due to coupling of graphene to a substrate ${ }^{12}$ and/or a gate field, the breaking of translational invariance due to impurities, ${ }^{13-15}$ localized states, ${ }^{16}$ and resonant coupling to extrinsic magnetic moments. ${ }^{17}$ In the case of nonlocal spin valves, the relaxation due the electronic coupling to magnetic electrodes is also being considered. ${ }^{10,11}$

Here we take the opposite point of view and we consider intrinsic spin relaxation in graphene due to the interplay between its unique mechanical and electronic properties. We show that flexural distortions, unavoidable in two dimensional crystals $^{18}$ in the form of either static ripples or out-of-plane phonons, induce spin scattering between the Dirac electrons, to linear order in the flexural field. This coupling differs from the spin-conserving second-order interaction between Dirac electrons and flexural distortions ${ }^{19}$ that has been proposed as an intrinsic limit to mobility in suspended high quality samples. ${ }^{20,21}$

The fact that curvature enhances spin-orbit scattering has been discussed ${ }^{22}$ and observed ${ }^{23}$ in the case of carbon nanotubes. Local curvature is also expected to enhance spin orbit in graphene ${ }^{24-27}$ and graphene ribbons. ${ }^{28}$ In the present paper we derive a microscopic Hamiltonian that describes explicitly the spin-flip scattering of electronic states of graphene due to both dynamic and static flexural distortions. We describe graphene by means of a multiorbital atomistic description that naturally accounts for the two crucial ingredients of the proposed intrinsic spin-phonon coupling: the intra-atomic spin-orbit coupling (SOC) and the modulation of the interatomic integrals due to atomic displacements. Importantly, both the SOC and the flexural distortions couple the Dirac electrons to higher energy $\sigma$ bands, in the spin-flip and spin conserving channels, respectively. Their combined action results in an effective spin-flip interaction for the Dirac electrons.

The spin-flip lifetimes computed from our theory are in the range of $\mu$ s at room temperature. Therefore, the observation of spin lifetimes in the nanosecond regime implies that other extrinsic spin relaxation mechanisms are effective. Our results provide an upper limit for the lifetime that will be relevant when graphene samples can be prepared without extrinsic sources of spin relaxation. In the case of the proposed intrinsic spin relaxation mechanism, we find that the spin lifetime of electrons depends crucially on the long wavelength mechanical properties of the sample, determined by its coupling to the environment. We also find that the spin relaxation lifetime depends on the quantization axis along which the spin scattering is taking place as well as on the relative angle between the electron crystal momentum $\vec{k}$ and the crystal lattice.

\section{MICROSCOPIC MODEL}

Our starting point is the tight-binding Hamiltonian $\mathcal{H}=$ $\mathcal{H}_{\mathrm{SC}}+\mathcal{H}_{\mathrm{SOC}}$ for electrons with spin $s$ moving in a lattice of atoms $\vec{r}$, with atomic orbitals $o$. We write the hopping part as

$$
\mathcal{H}_{\mathrm{SC}}=\sum_{\vec{r}, \vec{r}^{\prime}, o, o^{\prime}, s} H_{o, o^{\prime}}\left(\vec{r}-\vec{r}^{\prime}\right) \Psi_{\vec{r}, o, s}^{\dagger} \Psi_{\vec{r}^{\prime}, o^{\prime}, s},
$$

considering explicitly the dependence of the (spin conserving) interatomic matrix elements on the positions of the atoms. The intra-atomic SOC reads

$$
\mathcal{H}_{\mathrm{SOC}}=\sum_{\vec{r}, o, o^{\prime}, s, s^{\prime}} \lambda\left\langle\vec{r} o \sigma|\vec{L}(\vec{r}) \cdot \vec{S}| \vec{r} o^{\prime} s^{\prime}\right\rangle \Psi_{\vec{r}, o, s}^{\dagger} \Psi_{\vec{r}, o^{\prime}, s^{\prime}},
$$


where $\vec{S}$ is the spin operator, $\vec{L}(\vec{r})$ is the orbital angular momentum operator acting upon the atomic orbitals of site $\vec{r}$, and $\lambda$ is the spin-orbit coupling parameter.

Deviations from the ideal graphene lattice affect its electronic properties via modifications of the transfer integrals in Eq. (1). Their dependence on the interatomic distance, for example, gives rise to an electron-phonon interaction analogous to that of conducting polymers ${ }^{29}$ and leads to the appearance of effective gauge fields. ${ }^{25,30}$ In the present model, we consider instead the coupling with flexural distortions arising from the angular dependence of the interatomic Hamiltonian. We describe corrugations away from perfectly flat graphene in the form $\vec{r} \simeq \vec{r}_{0}+h(\vec{r}) \hat{z}$, where $\vec{r}_{0}$ is a vector of the honeycomb lattice and $h(\vec{r})$ is the displacement of atom $\vec{r}$ perpendicular to the graphene sheet. We expand the interatomic Hamiltonian matrix to lowest order in the flexural field $h(\vec{r})$ and rewrite the Hamiltonian as $\mathcal{H}=\mathcal{H}_{0}+\mathcal{V}$, where $\mathcal{H}_{0}$ now describes ideally flat graphene including the weak intra-atomic SOC perturbation, and

$\mathcal{V}=\sum_{r, r^{\prime}, o, o^{\prime}, s}\left[h(\vec{r})-h\left(\vec{r}^{\prime}\right)\right] \frac{\partial}{\partial z} H_{o, o^{\prime}}\left(\vec{r}_{0}-\vec{r}_{0}^{\prime}\right) \Psi_{\vec{r}, o, s}^{\dagger} \Psi_{\vec{r}^{\prime}, o^{\prime}, s}$

is the spin-conserving coupling between electrons and corrugations.

\section{ELECTRON-FLEXURAL PHONON SCATTERING}

It is now convenient to recast Eq. (3) in terms of the eigenstates of the Hamiltonian $\mathcal{H}_{0}$ for flat graphene. In this context, the atomic positions $\vec{r}=\vec{R}+\overrightarrow{r_{\alpha}}$, are specified by their unit cell vector, $\vec{R}$, and their position $\vec{r}_{\alpha}$ inside the cell (sublattice index $\alpha=A, B$ ). The creation operators for Bloch states are related to atomic orbitals through

$$
c_{v \vec{k}}^{\dagger}=\frac{1}{\sqrt{N}} \sum_{\vec{R}, \alpha, o, s} e^{i \vec{k} \cdot \vec{R}} \mathcal{C}_{\nu, \vec{k}}(\alpha, o, s) \Psi_{\vec{R}+\vec{r}_{\alpha}, o, s}^{\dagger},
$$

where $\vec{k}$ is the wave vector, the coefficients $\mathcal{C}_{v, \vec{k}}(\alpha, o, s)$ are obtained from the diagonalization of the Bloch matrix, and $v$ is an index that labels the resulting bands (with mixed spin and angular momentum). Similarly, we expand the flexural field on each sublattice in its Fourier components, $h_{\alpha}(\vec{R})=$ $\frac{1}{\sqrt{N}} \sum_{\vec{q}} e^{-i \vec{q} \cdot \vec{R}} h_{\alpha}(\vec{q})$. After a lengthy but straightforward calculation, we can express Eq. (3) as a term causing scattering between crystal states with different momentum and band indices:

$$
\begin{aligned}
\mathcal{V}= & \sum_{\vec{k}, \vec{k}^{\prime}, v, \nu^{\prime}} \mathcal{V}_{v, v^{\prime}}\left(\vec{k}, \vec{k}^{\prime}\right) c_{\nu, \vec{k}}^{\dagger} c_{\nu^{\prime}, \vec{k}^{\prime}}, \\
\mathcal{V}_{v, v^{\prime}}\left(\vec{k}, \vec{k}^{\prime}\right) \equiv & \frac{1}{\sqrt{N}} \sum_{\vec{R}, \alpha, \alpha^{\prime}, o, o^{\prime}, \sigma} \frac{\partial}{\partial z} H_{o, o^{\prime}}\left(\vec{r}_{\alpha}-\vec{r}_{\alpha^{\prime}}-\vec{R}\right) \\
& \times F_{\alpha \alpha^{\prime}}^{\vec{R}}\left(\vec{k}, \vec{k}^{\prime}\right) \mathcal{C}_{\nu, \vec{k}}^{*}(\alpha, o, \sigma) \mathcal{C}_{v^{\prime}, \vec{k}^{\prime}}\left(\alpha^{\prime}, o^{\prime}, \sigma\right) .
\end{aligned}
$$

The coupling is linear in the flexural phonon field, through the form factor $\left.F_{\alpha \alpha^{\prime}} \vec{R}, \vec{k}, \vec{k}^{\prime}\right)=h_{\alpha}\left(\vec{k}-\vec{k}^{\prime}\right) e^{i \vec{k}^{\prime} \cdot \vec{R}}-h_{\alpha^{\prime}}\left(\vec{k}-\vec{k}^{\prime}\right) e^{i \vec{k} \cdot \vec{R}}$. This should be contrasted with the electron-flexural phonon coupling usually considered within the $\pi$ subspace, ${ }^{19-21,30}$ that is quadratic in the field because of the quadratic dependence
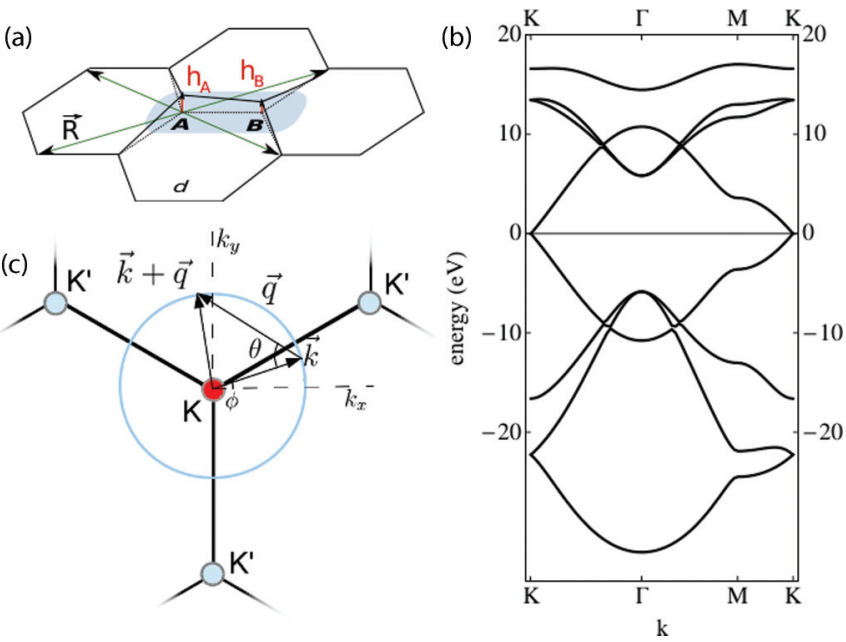

FIG. 1. (Color online) (a) Sketch of a local corrugation of the graphene layer. The shaded area is the unit cell. Green arrows indicate the interatomic contributions to the electron-flexural phonon coupling of Eq. (6). (b) The band structure resulting from our Slater-Koster parametrization. (c) Kinematics of the scattering process around the Dirac point $K$ in the low phonon frequency limit.

of interatomic distances on $h$. Note that $H_{o o^{\prime}}$ is short ranged within our tight-binding description, which limits $\vec{R}$ to the four vectors connecting neighboring cells (intercell coupling), as illustrated in Fig. 1(a), plus the null vector (intracell coupling).

\section{A. Slater-Koster parametrization}

Equations (5) and (6) provide a general recipe to compute the coupling of electrons of a generic tight-binding Hamiltonian to a flexural field. We now show that because we have included the SOC in the reference Hamiltonian $\mathcal{H}_{0}$, the perturbation Eq. (5) is able to induce a direct coupling between states with opposite spin. Following previous work, ${ }^{22,24,26,28,31}$ we consider a subset of four valence orbitals of the carbon atom, namely $o=s, p_{x}, p_{y}, p_{z}$, and adopt a Slater-Koster $(\mathrm{SK})^{32}$ parametrization for the tight-binding Hamiltonian Eq. (1) ${ }^{33,34}$ In this framework, the interatomic matrix elements $H_{o, o^{\prime}}$ connecting the atom $\alpha$ at $\vec{r}_{\alpha}$ with atom $\alpha^{\prime}$ at $\vec{r}_{\alpha^{\prime}}+R$ can be expressed in terms of four parameters, $V_{s s}, V_{s p}, V_{\sigma \pi}$, $V_{\pi \pi}$, taken from Ref. 36, describing interorbital overlaps in the $s, p$ basis, ${ }^{28,32}$ and the three director cosines $l, m, n$ of the interatomic bond vectors $\vec{\rho}=\vec{r}_{\alpha}-\vec{r}_{\alpha^{\prime}}-\vec{R}$, defined by $\vec{\rho} \equiv \rho(l \hat{x}+m \hat{y}+n \hat{z})$. Setting $U_{x} \equiv V_{p p \pi}+x^{2} V_{\sigma \pi}$ and $V_{\sigma \pi} \equiv\left(V_{p p \sigma}-V_{p p \pi}\right)$ we can write the SK matrix in compact form as

$$
H(\vec{\rho})=\left(\begin{array}{cccc}
V_{s s \sigma} & l V_{s p \sigma} & m V_{s p \sigma} & n V_{s p \sigma} \\
-l V_{s p \sigma} & U_{l} & \operatorname{lm} V_{\sigma \pi} & \ln V_{\sigma \pi} \\
-m V_{s p \sigma} & \operatorname{lm} V_{\sigma \pi} & U_{m} & m n V_{\sigma \pi} \\
-n V_{s p \sigma} & \ln V_{\sigma \pi} & m n V_{\sigma \pi} & U_{n}
\end{array}\right) .
$$

The unperturbed crystal states for flat graphene are described by $H(\vec{\rho})$ with $n=0$ (all bonds within the $x, y$ plane). The resulting band structure is shown in Fig. 1(b). From Eq. (6), the electron-flexural distortion coupling is determined by $\partial_{z} H(\vec{\rho})=(1 / d) \partial_{n} H(\vec{\rho})$, where $d$ is the equilibrium C-C distance. Direct inspection of Eq. (7) shows that $H(\vec{\rho})(n=0)$ does not couple the $p_{z}$ and $s, p_{x}, p_{y}$ sectors, while $\partial_{n} H(\vec{\rho})$ does. 
As a result, the $\pi$ and $\sigma$ bands of flat graphene are not mixed in the absence of SOC, unless they scatter with flexural distortions. In the presence of SOC, however, the low-energy $\pi$ states with spin $s$ mix with the $\sigma$ states with opposite spin already within the reference $\mathcal{H}_{0}$ for flat graphene. Close to the Dirac points, where the $\pi$ and $\sigma$ bands are separated in energy by a gap $E_{\sigma \pi}$, the spin $\pi-\sigma$ mixing is proportional to $\lambda / E_{\sigma \pi}$. Since this correction is small, the low energy Dirac bands of $\mathcal{H}_{0}$ can still be labeled according to their dominant spin character, that we denote as $\Uparrow$ and $\Downarrow$.

The above derivation shows that there are two perturbations that couple $\pi$ and $\sigma$ states: the spin-conserving coupling to the flexural field and the spin-flip SOC. Their combination is able to yield a spin-flip channel within the low energy $\pi$ bands, that is linear in both the flexural deformation and in the atomic spin-orbit coupling $\lambda$. This is very similar to the so called Rashba spin-orbit coupling, induced by the combination of $\pi-\sigma$ mixing due to an external electric field and atomic spin-orbit coupling, ${ }^{24,27,31}$ and different from the $\lambda^{2}$ scaling of the intrinsic SOC in flat graphene.

\section{B. Effective Hamiltonian}

We now apply the microscopic theory developed above to obtain the effective spin-flip Hamiltonian for electrons close to the Dirac points. As it turns out, the spin-flip scattering rate is proportional to the occupation of the phonon modes [Eqs. (9) and (12)]. Therefore, we consider only the lower flexural branch for which $\omega_{q} \propto q^{2}$ (see below), and discard the out of phase vibrations of the two sublattices, whose energy lies tens of $\mathrm{meV}$ above $\mathrm{e}^{37}$ and have an exponentially smaller occupation.

The flexural field is factored out from Eq. (5) by setting $h_{A}(\vec{q})=h_{\vec{q}}$ and $h_{B}(\vec{q})=e^{i \vec{q} \cdot\left(\vec{r}_{B}-\vec{r}_{A}\right)} h_{\vec{q}}$, which yields

$$
\mathcal{V}=\sum_{\vec{k}, \vec{q}, v, v^{\prime}} M_{\nu, v^{\prime}}(\vec{k}, \vec{q}) \frac{h_{\vec{q}}}{d} c_{\nu, \vec{k}+\vec{q}}^{\dagger} c_{v^{\prime}, \vec{k}},
$$

with $\mathcal{V}_{v, v^{\prime}}(\vec{k}+\vec{q}, \vec{k})=\frac{h_{\vec{q}}}{d} M_{v, v^{\prime}}(\vec{k}, \vec{q})$. The standard form for the phonon spin-flip interaction in second quantization is readily obtained by substituting $h_{\vec{q}}=\sqrt{\frac{\hbar}{2 M_{C} \omega_{\vec{q}}}}\left(a_{-\vec{q}}^{\dagger}+a_{\vec{q}}\right)$ into Eq. (8), with $M_{C}$ the carbon mass.

\section{Spin quantization axis}

In the case of systems with time reversal invariance and inversion symmetry, a Bloch state with momentum $\vec{k}$ has a twofold Kramers degeneracy. This is definitely the case of the ideal flat graphene. As a result, there are infinitely many possible choices of the pairs of degenerate states $v$ and $v^{\prime}$. In the calculations below we select a given pair by including in the Hamiltonian an external magnetic field along the direction $\hat{n}$ with magnitude negligible compared with all other energy scales in the problem, but enough to split the Kramers doublet and choose its spin quantization axis. Importantly, the effective electron-phonon coupling depends on this choice, i.e., it depends on $\hat{n}$. In the following we include $\hat{n}$ as an argument of the phonon spin-flip coupling and we label the two bands as $\Uparrow$ and $\Downarrow$, which are referred to the quantization axis $\hat{n}$. The fact that the phonon spin-flip coupling depends on $\hat{n}$ means that the strength of the spin-flip Hamiltonian is not isotropic in the spin space. This will lead to an anisotropy of the spin relaxation in graphene, closely related to the one recently proposed in the case of metals. ${ }^{38}$

\section{SPIN RELAXATION}

\section{A. Spin relaxation rates}

The spin relaxation rate can now be calculated from Eq. (8) via the Fermi golden rule. Because the dispersion of the flexural modes is much weaker than the electronic dispersion, we can safely neglect the phonon frequency in the energy conservation. The relaxation rate for an electron with momentum $\vec{k}$ in the band $\Uparrow$ is then obtained by summing over both phonon absorption and emission processes and over all possible final states in the $\Downarrow$ band, which yields

$$
\Gamma_{\vec{k}, \hat{n}}=\frac{2 \pi}{\hbar} \int \frac{d^{2} q}{(2 \pi)^{2}}\left|M_{\Uparrow, \Downarrow}(\vec{k}, \vec{q}, \hat{n})\right|^{2}\left\langle h_{\vec{q}}^{2}\right| \delta\left(E_{\vec{k}+\vec{q}}-E_{\vec{k}}\right) .
$$

This, together with the explicit expressions for the spin-flip matrix elements in Eq. (6), constitutes the main result of this work. From Eq. (9) it is clear that once the specific form of the electron-flexural phonon coupling, $M_{\Uparrow, \downarrow}(\vec{k}, \vec{q}, \hat{n})$, is known, the behavior of the spin relaxation rate is fully determined by the statistical fluctuations of the flexural field, $\left\langle h_{\vec{q}}^{2}\right\rangle$. Interestingly, the above expression describes on an equal footing both low-frequency flexural modes (that arise in free-standing or weakly bound graphene or graphite) as well as static ripples (relevant to graphene deposited on a substrate). The proposed spin relaxation mechanism, therefore, applies without distinction to both physical situations.

For actual calculations we approximate the $\pi$ band energies as $E_{\vec{k}}= \pm \hbar v_{F} k$, which is valid except for a negligible interval around the Dirac point, where the SOC opens a gap of the order of a few $\mu \mathrm{eV}$. We have verified that trigonal warping corrections to this linear relation are smaller than $4 \%$ at the highest energy considered, $E_{F}=0.4 \mathrm{eV}$ (importantly, while a linear and isotropic approximation is used for the energy $E_{k}$, the wave functions that enter into the matrix elements $M_{\Uparrow, \Downarrow}$ retain the full symmetry of the honeycomb lattice). With this simplification, energy conservation implies $\sqrt{k^{2}+q^{2}+2 k q \cos \theta}=\sqrt{k^{2}}$. This fixes the relative angle between $\vec{k}$ and $\vec{q}$ to $\cos \theta=-\frac{q}{2 k}$; cf. Fig. 1(c). This equation has two solutions, that we label with the index $s$, which allows us to perform the angular integration in Eq. (9), yielding

$$
\Gamma_{\vec{k}, \hat{n}}=\sum_{s} \frac{1}{\pi \hbar} \int_{0}^{2 k} d q \frac{\left|M_{\Uparrow, \Downarrow}^{s}(k, q, \hat{n})\right|^{2}\left\langle h_{q}^{2}\right\rangle}{\hbar v_{F} \sqrt{1-(q / 2 k)^{2}}} .
$$

We see that only long-wavelength fluctuations contribute to the spin relaxation because energy conservation constrains the exchanged momentum to $q \leqslant 2 k$, with $k$ being a small momentum around the Dirac point. From our numerics, we find that in the relevant case of small $k$ and $q \rightarrow 0$, the matrix elements evaluated on the energy-conserving surface (i.e., on shell, where $q / 2 k=-\cos \theta$ ) satisfy

$$
\sum_{s}\left|M_{\Uparrow, \Downarrow}^{s}(k, \hat{n})\right|^{2} \approx c^{2}(\phi, \hat{n}) \lambda^{2} q^{4} d^{4},
$$




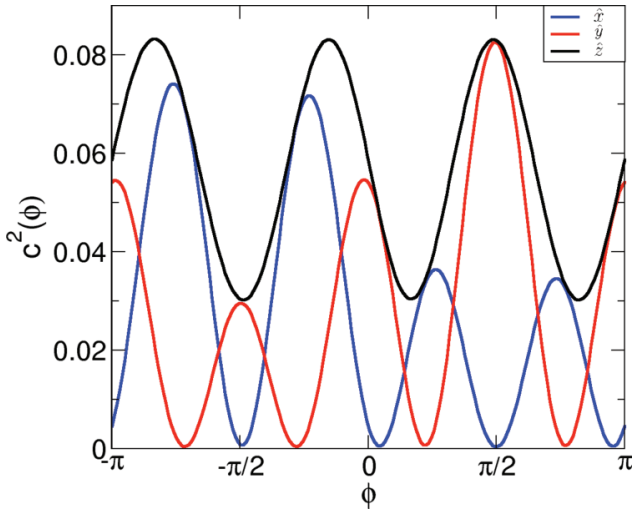

FIG. 2. (Color online) Spin-flip matrix elements as a function of the initial state wave-vector orientation $\phi$, for three different orientations of the spin quantization axis, $\hat{n}=\hat{x}, \hat{y}, \hat{z}$.

where $\phi$ is the angle formed between $\vec{k}$ and the $x$ axis in reciprocal space and $c(\phi, \hat{n})$ is a dimensionless coefficient that only depends on the angle $\phi$, the spin quantization axis, and the SK parameters. We plot this coefficient in Fig. 2, for three different orientations of the spin quantization axis. It can be checked numerically that $c^{2}(\phi, \hat{z})=c^{2}(\phi, \hat{x})+c^{2}(\phi, \hat{y})$. As a consequence of the angular dependence of $c(\phi)$, the relaxation rate evaluated from Eq. (10) is also angular dependent, as shown below.

\section{B. Fluctuations of the flexural field}

The scattering rate Eq. (10) depends on the statistical fluctuations of the flexural field $\left\langle h_{\vec{q}}^{2}\right\rangle$, which we evaluate here for different scenarios.

We start with the expression for free-standing graphene at thermal equilibrium:

$$
\left\langle h_{q}^{2}\right\rangle=\frac{\hbar}{2 M_{C} \omega_{q}}\left[1+2 n_{B}\left(\omega_{q}\right)\right] \simeq \frac{k_{B} T}{M_{C} \omega_{q}^{2}},
$$

where $n_{B}\left(\omega_{q}\right)$ is the thermal population of mode $q$ and the second equality holds when $k_{B} T \gg \hbar \omega_{q}$. For purely harmonic flexural modes, for which $\omega_{q} \simeq D q^{2}$, the fluctuation $\left\langle h_{\vec{q}}^{2}\right\rangle$ diverges as $q^{-4}$ for small $q$. When inserted into Eq. (10), this divergence exactly compensates the $q^{4}$ dependence of the matrix element Eq. (11). In real samples, however, the singularity of low-wavelength fluctuations is renormalized due to the interaction with other phonons (i.e., by anharmonic effects), ${ }^{39}$ and can be further cut off by the presence of strain ${ }^{21}$ or pinning to a substrate. ${ }^{40}$ The resulting dispersion can be parametrized as $\omega_{q}=D \sqrt{q^{4}+q^{4-\eta} q_{c}^{\eta}}$ for $\eta>0$ so that, in the long wavelength limit, ${ }^{39,41}$

$$
\left\langle h_{q}^{2}\right\rangle \propto \frac{1}{q^{4-\eta} q_{c}^{\eta}},
$$

where $\eta$ and $q_{c}$ depend on the physical mechanism of renormalization. Specifically, substrate pinning opens a gap in the phonon spectrum, ${ }^{40}$ corresponding to $\eta=4$; strain makes the dispersion linear at long wavelengths ${ }^{21}(\eta=2)$; anharmonic effects yield $\eta=0.82 .{ }^{39}$ Substrate roughness also gives rise to fluctuations in the form of Eq. (13), with $\eta=1.41,42$
Finally, from Eqs. (10) and (12) it can be argued that highenergy phonons connecting different Dirac cones $\mathrm{K}$ and $\mathrm{K}^{\prime}$ can be neglected, because the scattering rate is exponentially small for $k_{B} T \ll \hbar \omega_{q}$ (with $\hbar \omega_{q} \sim 0.1 \mathrm{eV}$; see Ref. 37).

\section{RESULTS AND DISCUSSION}

\section{A. Approximate estimate of the rate}

Before we discuss the results of our numerical integration of Eq. (10) it is convenient to obtain an approximate analytical formula from the integration of the small $q$ part. For that matter, we make use of the long wavelength expression Eq. (11), drop the square root factor in the denominator, which is only relevant in a very narrow region around the backscattering condition $q \approx 2 k$, and use the asymptotic expression Eq. (13) for flexural fluctuations. The approximate expression for the rate reads

$$
\Gamma_{\vec{k}_{F}, \hat{n}} \simeq \frac{d}{\pi \hbar} \frac{\lambda^{2} c(\phi, \hat{n}, \tau)}{\hbar v_{F}} \frac{\left(2 k_{F} d\right)^{\eta+1}}{(\eta+1)\left(q_{c} d\right)^{\eta}} r^{2}(T),
$$

which is valid at densities such that $k_{F} \ll q_{c}$. Here we have defined $r^{2}(T)=\frac{k_{B} T d^{2}}{M_{C} D^{2}} \frac{1}{d^{2}}$, representing the ratio between the short-range flexural fluctuations [i.e., Eq. (12) evaluated at $q=1 / d]$ and the interatomic distance $d$. From Eqs. (12) and (14) we see that in ideal graphene with $\eta=0$ the spin relaxation rate increases linearly with temperature, following the thermal population of flexural phonons. A weaker temperature dependence, $\Gamma_{k_{F}, n} \propto T^{1-\eta / 2}$, arises when anharmonic effects dominate, because the anharmonic cutoff is itself temperature dependent, $q_{c} \propto \sqrt{T}$. 18,39

Equation (14) permits a quick estimate of the efficiency of the spin rate. We see that for a given value of $q_{c}$, the spin lifetime increases as the exponent $\eta$ increases. A lower limit for the relaxation time is therefore obtained by setting $\eta=0$ which, for $\lambda=8 \mathrm{meV}, c=10^{-1}, v_{F}=10^{-6} \mathrm{~ms}^{-1}$, and $r^{2} \simeq 10^{-2}$, yields a lifetime $\tau_{s}=1 / \Gamma_{\vec{k}_{F}}$ on the order of $1 \mu \mathrm{s}$ at a density $n=10^{12} \mathrm{~cm}^{-2}$ and at room temperature. Lifetimes in the $\mu$ s range are also obtained in the case of static ripples arising from the roughness of the underlying substrate, as we have checked using the values of $r^{2}$ and $q_{c}$ deduced from the height profiles in Ref. 42. In that case the lifetime is temperature independent.

\section{B. Energy dependence of the intrinsic spin relaxation}

We now compute Eq. (10) numerically, without analytical approximations. The spin lifetime $\tau_{s}=1 / \Gamma_{k_{F}, \hat{n}}$ so obtained for electrons at the Fermi level is plotted in Figs. 3 and 4.

In Fig. 3 we show the the spin lifetime as a function of $E_{F}=\hbar v_{F} k_{F}$, fixing the momentum direction $\phi=0$, the valley $K$ [cf. Fig. 1(c)], and taking as spin quantization axis the off-plane direction $\hat{n}=\hat{z}$. We take ${ }^{21} D=4.6 \times 10^{-7} \mathrm{~m}^{2} \mathrm{~s}^{-1}$, $\lambda=8 \mathrm{meV},{ }^{23} T=300 \mathrm{~K}$, and $v_{F}=1.16 \times 10^{6} \mathrm{~m} / \mathrm{s}$ from our SK band structure. In each panel of Fig. 3, different curves correspond to different values of the scaling exponent $\eta$, i.e., to physically different mechanical environments for graphene. Panels (a) and (b) correspond to two different values of the cutoff momentum $q_{c}$. Two representative values, (a) $q_{c}=0.01 \AA^{-1}$ and (b) $q_{c}=0.1 \AA^{-1}$, are considered, covering the large spread of $q_{c}$ values available in the literature. In both panels, the result for ideal graphene in the harmonic 

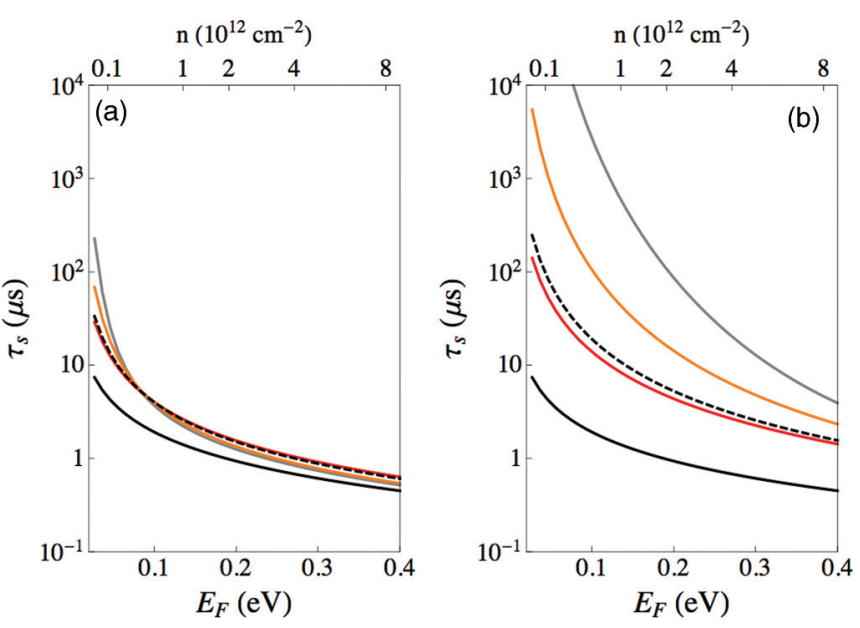

FIG. 3. (Color online) Room temperature spin lifetime calculated for electrons at the Fermi energy with momentum parallel to the $x$ axis ( $\phi=0$ ), for two different values of cutoff momentum: (a) $q_{c}=0.01$ $\AA^{-1}$ and (b) $q_{c}=0.1 \AA^{-1}$ (right) and different long-wavelength scaling laws: ideal graphene (black, $\eta=0$ ), including anharmonic effects (red, $\eta=0.82$ ), and including strain (orange, $\eta=2$ ) and substrate pinning (gray, $\eta=4$ ). The black dashed line is for $\eta=1$, which is representative for substrate roughness.

approximation is shown for reference (black) as it provides an absolute lower bound to the actual lifetime, in agreement with the estimate $\tau_{s} \sim 1 \mu$ s given after Eq. (14). Comparing Figs. 3(a) and 3(b) we see that the effect of the mechanical environment becomes more pronounced for large values of the cutoff momentum $q_{c}$. Because the spin relaxation is dominated by the low energy fluctuations of the membrane, the shortest spin lifetimes, excluding the harmonic theory, are obtained in
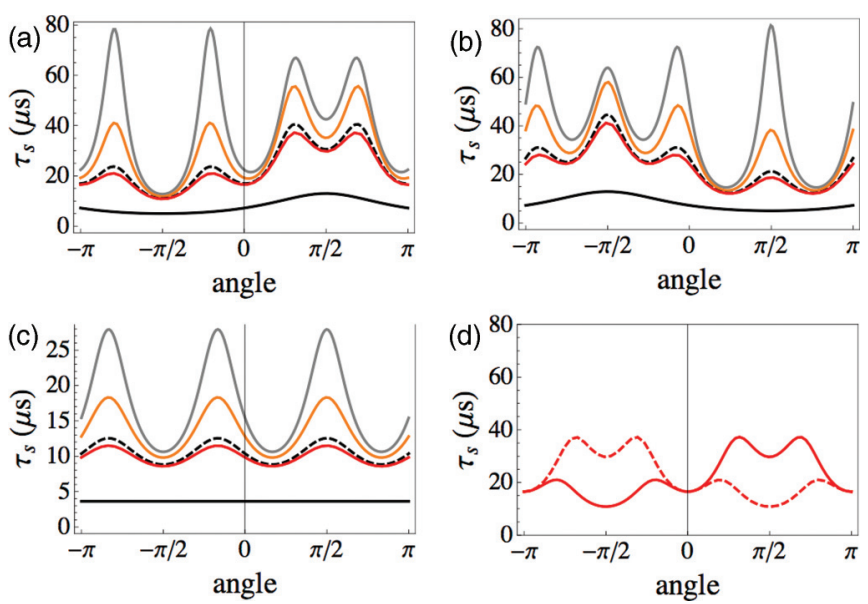

FIG. 4. (Color online) Spin lifetime as a function of $\phi$, the polar angle of the momentum of the initial state, at room temperature, calculated for $E_{F}=54 \mathrm{meV}$, momentum cutoff $q_{c}=0.01 \AA^{-1}$, for quantization axis $\hat{n}=\hat{x}$ (a), $\hat{y}$ (b), and $\hat{z}$ (c), and different longwavelength scaling laws: ideal graphene (black, $\eta=0$ ), including anharmonic effects (red, $\eta=0.82$ ), and including strain (orange, $\eta=2$ ) and substrate pinning (gray, $\eta=4$ ). The black dashed line is for $\eta=1$, which is representative for substrate roughness. Panel (d): showing the results for the anharmonic case $\eta=0.82$ and quantization axis $\hat{n}=\hat{x}$ [i.e., same as in (a)] with the initial state in two different valleys. suspended unstrained graphene (red curve), i.e., when external mechanical influences are minimized and the mobility is possibly largest. Even longer spin lifetimes can in principle be achieved by suppressing the fluctuations of the graphene membrane, by an applied strain (orange) or substrate pinning (gray). Pinning by interlayer binding forces should also inhibit the spin relaxation in epitaxially grown graphene.

For all the mechanical models considered here, the spin relaxation time is a decreasing function of the density, because the phase space for spin-flip scattering increases with $k_{F}$. The density dependence within the different models can be anticipated by substituting the Fermi wave vector $k_{F}=\sqrt{\pi n}$ in the analytical expression Eq. (14), which results in $\tau_{s} \propto$ $n^{-(\eta+1) / 2}$ for $k_{F} \lesssim q_{c}{ }^{43}$

Finally, it is apparent that in all instances the computed lifetimes are larger than 500 ns. Therefore, the proposed intrinsic spin relaxation mechanism cannot account for present experimental observations where the spin lifetime is in the nanosecond range, which are presumably dominated by other (extrinsic) relaxation mechanisms.

\section{Anisotropy}

We now consider the influence of the momentum orientation, the spin quantization axis, and the valley on the spin relaxation time. The results of Fig. 3 have been obtained for $\phi=0, \hat{n}=\hat{z}$, and valley K [cf. Fig. 1(c)]. We find that the spin relaxation lifetime of a state with momentum $\vec{k}$ depends on the angle $\phi$ formed between $\vec{k}$ and the $\hat{x}$ direction, the spin quantization axis, and the valley. Results for the angular and valley dependence are shown in Fig. 4 for $E_{F}=54 \mathrm{meV}$. Let us consider first $\hat{n}=\hat{z}$, Fig. 4(c). In this case the $\phi$ dependence shows $C_{3}$ rotation symmetry, dephased with respect to the one of the lattice. The momentum-direction anisotropy is not a small effect, as the lifetime changes by more than a factor 2 between maxima and minima, for both values of $E_{F}$. The curves $\tau_{s}(\phi)$ also depend on the spin quantization axis. The effective spin-orbit coupling Hamiltonian ${ }^{31}$ for ideal flat graphene is proportional to the product of the spin and valley operators. Therefore, it is not surprising that the spin relaxation is different for $\hat{n}$ in plane and off plane. We have verified that $120^{\circ}$ rotations in the plane leave the spin lifetime unchanged, unlike the $90^{\circ}$ rotation necessary to go from $\hat{n}=\hat{x}$ to $\hat{n}=\hat{y}$ [Figs. 4(a) and 4(b)]. In general, we find that spin lifetimes are a factor of 2 to 3 longer for spin quantization axis in the plane than off plane. In a spin injection experiment $\hat{n}$ would be fixed by the magnetization orientation of the spin injector. Present experimental results show the opposite trend, ${ }^{3}$ which is a further indication that other extrinsic mechanisms are dominant.

The curves in Figs. 4(a)-4(c) are asymmetric in the sense that $\int_{-\pi}^{0} \tau(\phi) d \phi \neq \int_{0}^{\pi} \tau_{s}(\phi) d \phi$, where $\tau_{s}$ is computed for a given valley. Interestingly, the symmetry is restored when summing over the two valleys, as shown in Fig. 4(d). In particular, we find the expected relation:

$$
\tau_{s}(\phi, K)=\tau_{s}\left(-\phi, K^{\prime}\right) .
$$

This is a consequence of time-reversal symmetry, which imposes that wave functions of states in different valleys have opposite polar angles. 
Altogether, the results of Fig. 4 show that the spin relaxation time due to scattering with flexural distortions is anisotropic on three counts: spin quantization axis, valley, and momentum direction. Future work will determine if the preferred drift along a given direction, determined by an in-plane electric field, together with an externally imposed spin polarization, can serve to generate an imbalance in the valley occupations, and thereby an orbital magnetization in graphene. ${ }^{44}$

\section{CONCLUDING REMARKS}

In summary, we have shown that corrugations, that are ubiquitous in graphene in the form of dynamical flexural phonons or static ripples, enable a direct spin-flip mechanism to linear order in both the flexural field and in the spin-orbit coupling. This mechanism provides an unavoidable source of spin relaxation that will set the upper limit for spin lifetimes once the extrinsic sources of spin relaxation that prevail in state of the art experiments are removed. Such a limit is however nonuniversal, as its precise value depends on graphene's mechanical environment that determines the long-wavelength behavior of the flexural field. At room temperature, intrinsic spin lifetimes in the microsecond range are expected in a very wide range of situations. Importantly, the intrinsic spin relaxation time of electrons in graphene shows a marked dependence on their momentum direction, valley, and spin quantization axis.

Finally, whereas the existence of an upper limit for spin lifetimes in graphene might present in the future an obstacle for certain applications such as spin transistors, the intrinsic spinlattice coupling could open the way for hybrid devices, where a confined vibrational phonon could be coupled resonantly to the spin-flip transitions of Zeeman split confined Dirac electrons. Microwave pumping of such a system could result in a maser behavior of the phonon mode. ${ }^{45}$

\section{ACKNOWLEDGMENTS}

This work has been financially supported by MEC-Spain (Grants No. FIS2010-21883-C02-01, No. FIS2009-08744, and No. CONSOLIDER CSD2007-0010) and European Union, as well as Generalitat Valenciana, grant Prometeo 2012-11.
*Permanent address: Departamento de Fisica Aplicada, Universidad de Alicante, Spain.

${ }^{1}$ B. Trauzettel, D. V. Bulaev, D. Loss, and G. Burkard, Nature Phys. 3, 192 (2007).

${ }^{2}$ N. Tombros, J. Csaba, M. Popinciuc, J. Mihaita, H. T. Jonkman, and B. J. van Wees, Nature (London) 448, 571 (2007).

${ }^{3}$ N. Tombros, S. Tanabe, A. Veligura, C. Jozsa, M. Popinciuc, H. T. Jonkman, and B. J. van Wees, Phys. Rev. Lett. 101, 046601 (2008).

${ }^{4}$ K. Pi, W. Han, K. M. McCreary, A. G. Swartz, Y. Li, and R. K. Kawakami, Phys. Rev. Lett. 104, 187201 (2010).

${ }^{5}$ T.- Y. Yang, J. Balakrishnan, F. Volmer, A. Avsar, M. Jaiswal, J. Samm, S. R. Ali, A. Pachoud, M. Zeng, M. Popinciuc, G. Guntherodt, B. Beschoten, and B. Ozyilmaz, Phys. Rev. Lett. 107, 047206 (2011).

${ }^{6}$ W. Han and R. K. Kawakami, Phys. Rev. Lett. 107, 047207 (2011).

${ }^{7}$ A. Avsar et al., Nano Lett. 11, 2363 (2011).

${ }^{8}$ W. Han, K. M. McCreary, K. Pi, W. H. Wang, Y. Li, H. Wen, J. R. Chen, and R. K. Kawakami, J. Magn. Magn. Mater. 324, 369 (2012).

${ }^{9}$ M. B. Lundeberg, R. Yang, J. Renard, and J. A. Folk, Phys. Rev. Lett. 110, 156601 (2013).

${ }^{10}$ T. Maassen, I. J. Vera-Marun, M. H. D. Guimaraes, and B. J. van Wees, Phys. Rev. B 86, 235408 (2012).

${ }^{11}$ F. Volmer, M. Drögeler, E. Maynicke, N. von den Driesch, M. L. Boschen, G. Güntherodt, and B. Beschoten, arXiv:1305.6484.

${ }^{12}$ C. Ertler, S. Konschuh, M. Gmitra, and J. Fabian, Phys. Rev. B 80, 041405(R) (2009).

${ }^{13}$ A. H. Castro Neto and F. Guinea, Phys. Rev. Lett. 103, 026804 (2009).

${ }^{14}$ H. Ochoa, A. H. Castro Neto, and F. Guinea, Phys. Rev. Lett. 108, 206808 (2012).

${ }^{15}$ D. V. Fedorov, M. Gradhand, S. Ostanin, I. V. Maznichenko, A. Ernst, J. Fabian, and I. Mertig, Phys. Rev. Lett. 110, 156602 (2013).
${ }^{16}$ T. Maassen, J. J. van den Berg, E. H. Huisman, H. Dijkstra, F. Fromm, T. Seyller, and B. J. van Wees, Phys. Rev. Lett. 110, 067209 (2013).

${ }^{17}$ D. Kochan, M. Gmitra, and J. Fabian, arXiv:1306.0230.

${ }^{18}$ A. Fasolino, J. H. Los, and M. I. Katsnelson, Nat. Mater. 6, 858 (2007).

${ }^{19}$ E. Mariani and F. von Oppen, Phys. Rev. Lett. 100, 076801 (2008).

${ }^{20}$ S. V. Morozov, K. S. Novoselov, M. I. Katsnelson, F. Schedin, D. C. Elias, J. A. Jaszczak, and A. K. Geim, Phys. Rev. Lett. 100, 016602 (2008).

${ }^{21}$ E. V. Castro, H. Ochoa, M. I. Katsnelson, R. V. Gorbachev, D. C. Elias, K. S. Novoselov, A. K. Geim, and F. Guinea, Phys. Rev. Lett. 105, 266601 (2010).

${ }^{22}$ T. Ando, J. Phys. Soc. Jpn. 69, 1757 (2000).

${ }^{23}$ F. Kuemmeth, S. Ilani, D. C. Ralph, and P. L. McEuen, Nature (London) 452, 448 (2008).

${ }^{24}$ D. Huertas-Hernando, F. Guinea, and A. Brataas, Phys. Rev. B 74, 155426 (2006).

${ }^{25}$ D. Huertas-Hernando, F. Guinea, and A. Brataas, Phys. Rev. Lett. 103, 146801 (2009).

${ }^{26}$ J.-S. Jeong, J. Shin, and H.-W. Lee, Phys. Rev. B 84, 195457 (2011).

${ }^{27}$ V. K. Dugaev, E. Ya. Sherman, and J. Barnaś, Phys. Rev. B 83, 085306 (2011)

${ }^{28}$ D. Gosálbez-Martínez, J. J. Palacios, and J. Fernández-Rossier, Phys. Rev. B 83, 115436 (2011).

${ }^{29}$ W. P. Su, J. R. Schrieffer, and A. J. Heeger, Phys. Rev. Lett. 42, 1698 (1979).

${ }^{30}$ A. H. Castro Neto, F. Guinea, N. M. R. Peres, K. S. Novoselov, and A. K. Geim, Rev. Mod. Phys. 81, 109 (2009).

${ }^{31}$ H. Min, J. E. Hill, N. A. Sinitsyn, B. R. Sahu, L. Kleinman, and A. H. MacDonald, Phys. Rev. B 74, 165310 (2006).

${ }^{32}$ J. C. Slater and G. F. Koster, Phys. Rev. 94, 1498 (1954). 
${ }^{33}$ In the calculations we have left out $d$ orbitals, for simplicity. It has been shown that they give the dominant contribution to the SOC gap at the Dirac points, ${ }^{34,35}$ but they do not renormalize the change of the gap with the flexural distortion, ${ }^{34}$ which is related to the spin-phonon coupling proposed here. In any event, our results provide a lower limit for the instrinsic spin relaxation mechanism, given the fact that including the $d$ orbitals will enhance the effect.

${ }^{34}$ M. Gmitra, S. Konschuh, C. Ertler, C. Ambrosch-Draxl, and J. Fabian, Phys. Rev. B 80, 235431 (2009).

${ }^{35}$ S. Konschuh, M. Gmitra, and J. Fabian, Phys. Rev. B 82, 245412 (2010).

${ }^{36}$ D. Porezag, Th. Frauenheim, Th. Kõhler, G. Seifert, and R. Kaschner, Phys. Rev. B 51, 12947 (1995).

${ }^{37}$ R. Saito, G. Dresselhaus, and M. S. Dresselhaus, Physical Properties of Carbon Nanotubes (Imperial College Press, London, 1998).
${ }^{38}$ B. Zimmermann, P. Mavropoulos, S. Heers, N. H. Long, S. Blügel, and Y. Mokrousov, Phys. Rev. Lett. 109, 236603 (2012).

${ }^{39}$ K. V. Zakharchenko, R. Roldán, A. Fasolino, and M. I. Katsnelson, Phys. Rev. B 82, 125435 (2010).

${ }^{40}$ J. Sabio, C. Seoánez, S. Fratini, F. Guinea, A. H. Castro Neto, and F. Sols, Phys. Rev. B 77, 195409 (2008).

${ }^{41}$ M. I. Katsnelson and A. K. Geim, Philos. Trans. R. Soc. A 366, 195 (2008).

${ }^{42}$ M. Ishigami, J. H. Chen, W. G. Cullen, M. S. Fuhrer, and E. D. Williams, Nano Lett. 7, 1643 (2007).

${ }^{43}$ This result is strictly valid for $k_{F} \gg k_{B} T / v_{F}$, which ensures that spin relaxation arises from a narrow interval around $k_{F}$.

${ }^{44}$ D. Xiao, W. Yao, and Q. Niu, Phys. Rev. Lett. 99, 236809 (2007).

${ }^{45}$ I. Bargatin and M. L. Roukes, Phys. Rev. Lett. 91, 138302 (2003). 